\title{
Open-air traveling-wave thermoacoustic generator
}

\author{
XIE XiuJuan ${ }^{1 *}$, GAO Gang ${ }^{1,2}$, ZHOU Gang $^{1} \&$ LI Qing $^{1}$ \\ ${ }^{1}$ Key Laboratory of Cryogenics, Technical Institute of Physics and Chemistry, Chinese Academy of Sciences, Beijing 100190, China; \\ ${ }^{2}$ Graduate University of Chinese Academy of Sciences, Beijing 100049, China
}

Received November 19, 2010; accepted February 15, 2011

\begin{abstract}
Taking the input and reflected waves into account, the relationship between the acoustic impedance at the end and the input of a system were theoretically analyzed. Closed and open acoustic configurations that influence the pressure, volumetric velocity, impedance and acoustic work were compared in detail. Based on the above investigation, an open-air traveling-wave thermoacoustic generator was designed and fabricated. It is composed of a looped tube, a resonator open at one end, a regenerator, and hot and cold heat exchangers. It is a small scale and simple configuration. The resonant frequency is $74 \mathrm{~Hz}$ at $1 \mathrm{bar}$ in air. The maximum acoustic pressures at the open end and $0.5 \mathrm{~m}$ far away from the open end are $133.4 \mathrm{~dB}$ and $101 \mathrm{~dB}$ from a reference value of $20 \mu \mathrm{Pa}$ when the heating power was $210 \mathrm{~W}$, respectively. Acoustic pressure is reasonable for practical application as a low-frequency acoustic source. In further work, we believe that the acoustic pressure at the open end can achieve $150 \mathrm{~dB}$, which could be a solution to problems in existing acoustic generators. These problems include low acoustic pressure and system complexity. It can be used as a basic acoustic source for low frequency and long-range noise experiments, and as a supply for high acoustic pressures necessary for industrial sources.
\end{abstract}

open-air, traveling-wave, thermoacoustic generator

Citation: Xie X J, Gao G, Zhou G, et al. Open-air traveling-wave thermoacoustic generator. Chinese Sci Bull, 2011, 56: 2167-2173, doi: 10.1007/s11434-011$4427-\mathrm{x}$

The thermoacoustic principle [1] is a thermodynamic effect that occurs between compressible gases (the first medium) and solids (the second medium) in an acoustic field. It results in a time-averaged heat flow and a time-averaged work flow along (against) the sound propagation direction at penetration depths that are far from the solid boundaries. The conversion of heat to work is called thermal to acoustic effect, and the opposing process is called the acoustic to thermal effect. Based on these two effects, thermoacoustic systems can be classified as thermoacoustic engines and thermoacoustic refrigerators. A regenerator in an acoustic field can bring standing-waves [2,3], traveling-waves [4-6] and standing-traveling waves $[7,8]$ into being. In the latest two decades, engineering applications in thermoacoustic refrigeration and thermoacoustic electricity have been rapidly developed. In 1990, thermoacoustic engines first re-

*Corresponding author (email: xiexiujuan@mail.ipc.ac.cn) placed linear compressors to drive pulse tube refrigerators [9]. Researchers of China have focused on large-scale thermoacoustic engines to drive pulse tube refrigerators and high-frequency thermoacoustic systems since 2000 [10-12]. Recently, a new lowest cooling temperature in liquid hydrogen, $20 \mathrm{~K}$, was achieved [13]. In 2003, the thermoacoustic engine was applied in an electrical field to drive a linear AC generator [14]. Luo's groups [15] investigated thermoacoustic electricity prototypes and linear generators, which is capable of producing electric powers in the hundreds of watts.

For acoustic configurations, thermoacoustic components such as regenerators and heat exchangers can be inserted into a closed resonator to create many types of thermoacoustic engines. The closed configuration can be filled with gases at MPa mean pressures. The gas types that can be used are flexible, including nitrogen, helium, argon, and a He-Ar mixture. The highest mean pressure that has been 
used is 5.5 MPa [14]. However, only thermoacoustic components that can withstand these pressures can be used. Thermoacoustic powers generally scale with $p_{\mathrm{m}} a A$, which is a dimensionless reference. Hence, for a given pressure ratio $\left|p_{1}\right| / p_{\mathrm{m}}$, a high mean pressure and high acoustic speed of gases yield a high power per unit volume of the system. Light gases also have a high thermal conductivity, which leads to higher penetration depths and, consequently, larger regenerator and heat exchanger gaps. This allows for easier heat exchanger fabrication, which is advantageous for closed thermoacoustic engine applications [16]. However, the closed configuration is restrictive in the output of acoustic waves in open spaces. Therefore, some researchers have given their attention to developing other applications.

In 2010, Slaton [17] constructed an open-air standingwave thermoacoustic system. The maximum acoustic pressure radiated from the open end of their resonator corresponded to $81 \mathrm{~dB}$ Sound Pressure Level (SPL) ref $20 \mu \mathrm{Pa}$ (1 $\mu \mathrm{Pa}=10^{-6} \mathrm{~Pa}$ ) for an input electric power of $276 \mathrm{~W}$. He concluded that a higher power version of that device may be used as a continuous source at low frequency. The standing-wave thermoacoustic system is a simple configuration and allows for easy oscillation, because of the relatively low required onset temperature. However, theoretically, the thermal efficiency of this system is lower than that of a traveling-wave thermoacoustic system because the standing-wave system has an irreversible thermal cycle [16]. Therefore, the performance of this open standing-wave system is limited. Compared with an open standing-wave system, an open traveling-wave thermoacoustic system has a higher onset temperature and is more difficult to design. However, this system can be used to design as acoustic generator with higher power output, because of its Stirling thermal cycle. This can significantly increase the maximum acoustic pressure compared with Slaton's system.

Investigations into open-air thermoacoustic systems are in their initial phases. In addition, a comparable closed system has not been reported in the literature. Therefore, we propose an open-air traveling-wave thermoacoustic generator. In this paper, taking the input and reflected waves into account, a theoretical model on the acoustic impedance at the end and at the input of the system were derived by solving the acoustic propagation equation. Comparisons of a closed acoustic configuration with an open system while accounting for the influence of pressure, volumetric velocity, impedance and acoustic work were analyzed in detail.
An open-air traveling-wave thermoacoustic generator was designed and fabricated. Experimental measurements of the pressure waveforms, and acoustic pressures at the open end and at $0.1 \mathrm{~m}, 0.3 \mathrm{~m}$ and $0.5 \mathrm{~m}$ from the open end at different heating powers were performed. The acoustic pressures with and without a cone were compared. The maximum acoustic pressure at the open end of the resonator was 133.4 $\mathrm{dB}$ (ref $20 \mu \mathrm{Pa}$ ) for a heating power of $210 \mathrm{~W}$.

\section{Open-air traveling-wave thermoacoustic generator}

The acoustic configuration of the traditional traveling-wave thermoacoustic engine is composed of a looped tube and a closed resonator. Thermoacoustic components such as regenerators and heat exchangers are inserted into the looped tube as shown in Figure 1(a). We adopted an open resonator in our traveling-wave thermoacoustic generator. The acoustic wave propagates in the open-air space, which is shown in Figure 1(b). The essential difference between the closed and open acoustic configurations is the end of resonator. A closed thermoacoustic system has a stable boundary condition. Different mean pressures and gas types can be used and can be adjusted for performance optimization according to the needs of the intended application. An open thermoacoustic system is open to the atmosphere, meaning the operating parameters are limited because the system must operate at 1 bar in air. Therefore, in this paper, the differences between the closed and open acoustic configurations are emphasized.

\section{Theoretical comparisons of closed and an open acoustic configuration}

The thermoacoustics that occur in the regenerator of looped tube can be simplified and treated as an acoustic source to analyze the influence of the closed or an open acoustic configuration in the system. These configurations are shown are in Figure 1(a) and (b). The continuity of the pressure and the velocity are assured at the surface of the looped tube that is connected to the resonator. This surface, namely the input of the resonator, is set to be the origin of the $x$-coordinate. Here, the acoustic impedance is $Z_{\mathrm{a} 0}$. The length of resonator is $l$, and the acoustic impedance at the end of resonator is $Z_{\mathrm{al}}$. This simple acoustic configuration is shown in Figure 2.

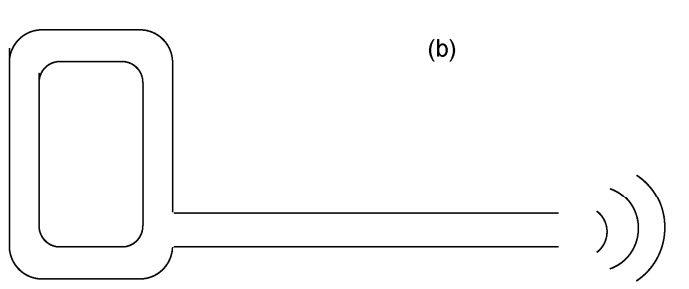

Figure 1 Schematic diagram of thermoacoustic system. (a) Closed traveling-wave thermoacoustic engine; (b) open-air traveling-wave thermoacoustic generator. 


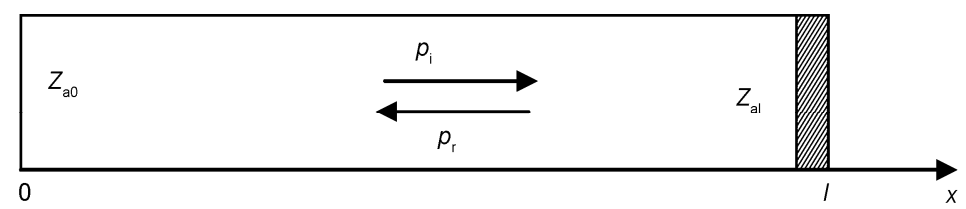

Figure 2 Simple model of acoustic configuration of the thermoacoustic system.

The work produced by the thermoacoustic effect in regenerator in the looped tube is transferred to the resonator. The pressure, $p_{1}$, can be decomposed into the input wave $p_{\mathrm{i}}$ and the reflected wave $p_{\mathrm{r}}$, because of the endcap of the resonator. $p_{\mathrm{i}}$ and $p_{\mathrm{r}}$ are expressed as $p_{\mathrm{i}}=p_{\mathrm{ai}} \mathrm{e}^{i(\omega t+k x)}$ and $p_{\mathrm{r}}=p_{\mathrm{ai}} \mathrm{e}^{i(\omega t-k x)}$, respectively. Here $\frac{p_{\mathrm{ar}}}{p_{\mathrm{ai}}}=r_{p}=\left|r_{p}\right| \mathrm{e}^{i \sigma \pi}, r_{p}$ is the reflected coefficient, $\sigma \pi$ is the phase difference at the interface of the input and reflected waves. Therefore, $p_{1}$ is defined as

$$
p_{1}=p_{\mathrm{i}}+p_{\mathrm{r}}=p_{\mathrm{ai}}\left[\mathrm{e}^{-i k x}+\left|r_{p}\right| \mathrm{e}^{i(k x+\sigma \pi)}\right] \mathrm{e}^{i \omega t}=\left|p_{1}\right| \mathrm{e}^{i(\omega t+\phi)},
$$

where pressure amplitude is $\left|p_{1}\right|=$ $p_{\text {ai }}\left|\sqrt{1+\left|r_{p}\right|^{2}+2\left|r_{p}\right| \cos 2 k\left(x+\sigma \frac{\lambda}{4}\right)}\right|$, and $\phi$ is the constant phase. According to the definition of the velocity, $v_{1}$ can be separated to $v_{\mathrm{i}}$ and $v_{\mathrm{r}}$, where $v_{\mathrm{i}}=v_{\mathrm{ai}} \mathrm{e}^{i(\omega t-k x)}=\frac{p_{\mathrm{ai}}}{\rho_{0} a_{0}} \mathrm{e}^{i(\omega t-k x)}$ and $v_{\mathrm{r}}=v_{\text {ar }} \mathrm{e}^{i(\omega t+k x)}=-\frac{p_{\mathrm{ar}}}{\rho_{0} a_{0}} \mathrm{e}^{i(\omega t+k x)}$. Therefore the velocity $v_{1}$ is given by

$$
v_{1}=v_{\mathrm{i}}+v_{\mathrm{r}}=\frac{p_{\mathrm{i}}}{\rho_{0} a_{0}}-\frac{p_{\mathrm{r}}}{\rho_{0} a_{0}} .
$$

Combining eqs. (1) and (2), the specific acoustic impedance along the resonator is found to be

$$
Z_{\mathrm{s}}=\frac{p_{1}}{v_{1}}=\rho_{0} a_{0} \frac{p_{\mathrm{ai}} \mathrm{e}^{-i k x}+p_{\mathrm{ar}} \mathrm{e}^{i k x}}{p_{\mathrm{ai}} \mathrm{e}^{-i k x}-p_{\mathrm{ar}} \mathrm{e}^{i k x}} .
$$

We can eliminate $p_{\mathrm{ai}}$ and $p_{\mathrm{ar}}$ by putting the specific acoustic impedances at $x=0$ and $x=l$ into eq. (3). Also, the specific acoustic impedance at $x=0$ can be expressed as one at $x=1$. From this we find

$$
Z_{\mathrm{s} 0}=\rho_{0} a_{0} \frac{Z_{\mathrm{sl}}+i \rho_{0} a_{0} \tan k l}{\rho_{0} a_{0}+i Z_{\mathrm{sl}} \tan k l}
$$

Therefore, the acoustic impedance at $x=0$ can be expressed as one at $x=1$ :

$$
Z_{\mathrm{a} 0}=\frac{\rho_{0} a_{0}}{A} \frac{Z_{\mathrm{al}}+i \frac{\rho_{0} a_{0}}{A} \tan k l}{\frac{\rho_{0} a_{0}}{A}+i Z_{\mathrm{al}} \tan k l},
$$

where $A$ is the cross-sectional area, $\rho_{0}$ and $a_{0}$ are the density and acoustic speed of the gas, respectively. $k=\frac{\omega}{a_{0}} \sqrt{\frac{1+(\gamma-1) f_{k} /\left(1+\varepsilon_{s}\right)}{1-f_{v}}}$ is complex wave number. $\omega$ and $\gamma$ are the angular frequency and ratio of isobaric and isochoric specific heats, respectively. Here, $f_{k}=\frac{(1+i) \delta_{k}}{2 r}$ and $f_{v}=\frac{(1+i) \delta_{v}}{2 r} \cdot r$ is the radius of the resonator. The thermal penetration depth, $\delta_{k}=\sqrt{\frac{2 k}{\omega \rho_{0} c_{p}}}=\sqrt{\frac{2 \kappa}{\omega}}$, and the viscous penetration depth, $\delta_{v}=\sqrt{\frac{2 \mu}{\omega \rho_{0}}}=\sqrt{\frac{2 v}{\omega}}$, indicate how far heat and momentum can diffuse laterally during a time interval on the order of the period of the oscillation divided by $\pi . \varepsilon_{s}=\left(\frac{k \rho_{0} c_{p}}{k_{s} \rho_{s} c_{s}}\right)^{1 / 2}$, and $k, \kappa, \mu, v$ and $c_{p}$ are the thermal conductivity, thermal diffusivity, dynamic diffusivity, kinematic viscosity, and isobaric heat capacity per unit mass of gas, respectively. $k_{s}, \rho_{s}$ and $c_{s}$ are the thermal conductivity, density and heat capacity per unit mass of solid, respectively.

(1) When the end has a closed endcap, $x=l, Z_{\mathrm{al}} \rightarrow \infty$, eq. (5) simplifies to

$$
Z_{\mathrm{a} 0} \approx-i \frac{\rho_{0} a_{0}}{A} \cot (k l) .
$$

Eq. (6) shows that the characteristics at the input the resonator are related to the value $k l$. The length of resonator is usually chosen to be in the range $l=(1 / 4-1 / 2) \lambda$ in the thermoacoustic system. Then, $k l=\frac{2 \pi}{\lambda} l \sqrt{\frac{1+(\gamma-1) f_{k} /\left(1+\varepsilon_{s}\right)}{1-f_{v}}}$ $\rightarrow(\pi / 2-\pi) \sqrt{\frac{1+(\gamma-1) f_{k} /\left(1+\varepsilon_{s}\right)}{1-f_{v}}}$, where $\lambda$ is the wavelength. The acoustic impedance is a function of the dimensions of the resonator, the mean pressure, the resonant frequency and the properties of the gas.

(2) When the end is open to air, for the condition of low frequency $k r<1$, the acoustic impedance at the end of the resonator is similar to the acoustic radiation of a limitless baffle plate. That is, $Z_{\mathrm{al}}=R_{\mathrm{al}}+i X_{\mathrm{al}}$, where the real part is $R_{\mathrm{al}} \approx \frac{\rho_{0} a_{0} k^{2} \pi r^{4}}{2}$, and the imaginary part is $X_{\mathrm{al}} \approx$ 
$\frac{8}{3} \rho_{0} a_{0} k r^{3}$. Eq. (5) can now be simplified to

$$
Z_{\mathrm{a} 0}=\frac{\rho_{0} a_{0}}{A} \frac{R_{\mathrm{al}}+i\left(X_{\mathrm{al}}+\frac{\rho_{0} a_{0}}{A} \tan k l\right)}{\left(\frac{\rho_{0} a_{0}}{A}-X_{\mathrm{al}} \tan k l\right)+i R_{\mathrm{al}} \tan k l} .
$$

Eqs. (6) and (7) show that the acoustic impedance at the input of the resonator is correlated with the dimensions of the resonator, the mean pressure, the resonant frequency and the properties of the gas. Therefore, for given resonator dimensions and a fixed resonant frequency, the differences between a closed and an open acoustic configuration can be quantitatively analyzed based on the linear thermoacoustic model [16]. For a closed acoustic configuration, the resonator has two parts, one is L1 with a length of $0.53 \mathrm{~m}$ and the other is L2 with a length of $1 \mathrm{~m}$, in which are filled with 1 bar and 5 bar of air, respectively. For an open acoustic configuration, a tube with a diameter of $2.5 \mathrm{~m}$ and a length of 1 $\mathrm{m}$ replaces L2. It has the same volume and length as a room. Then the end of L1 can be approximated as being open to the atmosphere. The operating parameters and properties of the gas are listed in Table 1 for the closed and open acoustic configurations. The frequency was maintained at $74 \mathrm{~Hz}$, and the variational range is about $3 \mathrm{~Hz}$ in the simulation. Therefore, the influence of frequency on the system can be ignored. From this, both the closed and open acoustic configurations were evaluated. The influence of the pressure, volumetric velocity, impedance and acoustic work were considered and are plotted in Figures 3(a), 3(b), 4 and 5.

The influences of the closed and open acoustic configu- rations on the pressure amplitude, $\left|p_{1}\right|$, and the volumetric velocity amplitude, $\left|U_{1}\right|$, along the length of the system are shown in Figure 3. When the end is closed, the minimum pressure amplitude occurs at $x=0.53 \mathrm{~m}$, not at the end of the resonator. The waveform of $\left|p_{1}\right|$ is about $3 / 4$ wavelength. The variational trend of $\left|U_{1}\right|$ is the opposite of $\left|p_{1}\right|$ and has it maximum at $x=0.53 \mathrm{~m}$. Also, it reaches zero at the end of resonator. The closed system can be filled with high pressure gas. $\left|p_{1}\right|$ is significantly enhanced at a mean pressure of 5 bar in air. However, $\left|U_{1}\right|$ decreases, which shows that the impedance increases and viscous losses decrease with $\left|U_{1}\right|$. When the end is open, the minimum value of $\left|p_{1}\right|$ is equal to zero and is at $x=0.53 \mathrm{~m}$. The waveform of $\left|p_{1}\right|$ is $1 / 4$ wavelength. $\left|U_{1}\right|$ in the open system is similar to that in the closed system. Compared with the closed acoustic configuration, the open configuration at same mean pressure can achieve a higher $\left|p_{1}\right|$ and $\left|U_{1}\right|$. However, $\left|p_{1}\right|$ is significantly lower and $\left|U_{1}\right|$ much greatly higher when the open configuration is compared to the closed with 5 bar air. Note that the acoustic pressure at the end of the resonator should be high for applications, but it is at its minimum value. This contradiction comes into being from two different mechanisms, and it is the key to allow the open system to significantly improve the acoustic pressure at the end of resonator.

Figure 4 shows the impedance amplitude, $\left|Z_{1}\right|$, along the length in the closed and open acoustic configurations. For the closed acoustic configuration, $\left|Z_{1}\right|$ reaches a maximum between $10^{9}-10^{10}$ at the end of resonator. This is caused by the antinode of $\left|p_{1}\right|$ and node of $\left|U_{1}\right| \cdot\left|Z_{1}\right|$ initially decreases and then increases, after reaching a minimum at $x=0.53 \mathrm{~m}$. For the open acoustic configuration, $\left|Z_{1}\right|$ has the maximum of $10^{6}$ at the input and the decreases to zero at the open end.

Table 1 Operating parameters and gas properties (ambient temperature $300 \mathrm{~K}$ )

\begin{tabular}{|c|c|c|c|c|c|}
\hline Acoustic configuration & Gas type & Mean pressure (bar) & Frequency $(\mathrm{Hz})$ & Thermal penetration depth $(\mu \mathrm{m})$ & Viscous penetration depth $(\mu \mathrm{m})$ \\
\hline \multirow{2}{*}{ Closed end } & air & 1 & 77.36 & 304.2 & 255.8 \\
\hline & air & 5 & 76.605 & 136.7 & 115 \\
\hline Open end & air & 1 & 74.1 & 334 & 284.2 \\
\hline
\end{tabular}
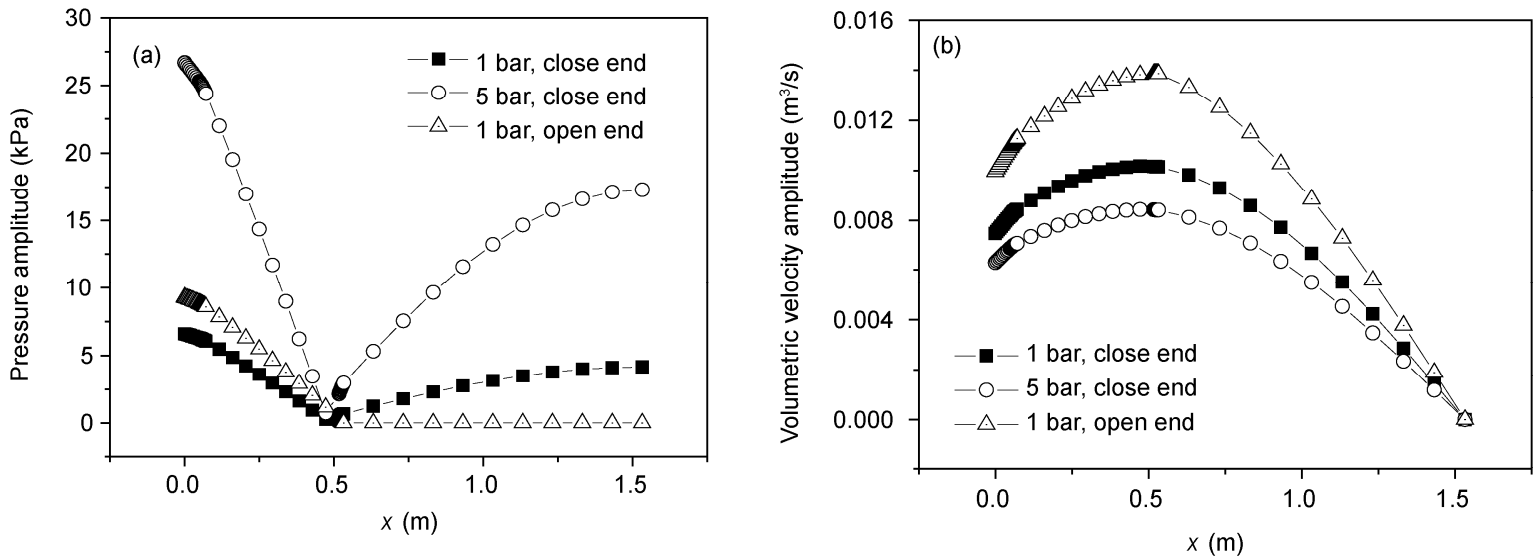

Figure 3 Pressure amplitude and volumetric velocity amplitude along the length in the closed and open acoustic configuration. (a) Pressure amplitude; (b) volumetric velocity amplitude. 


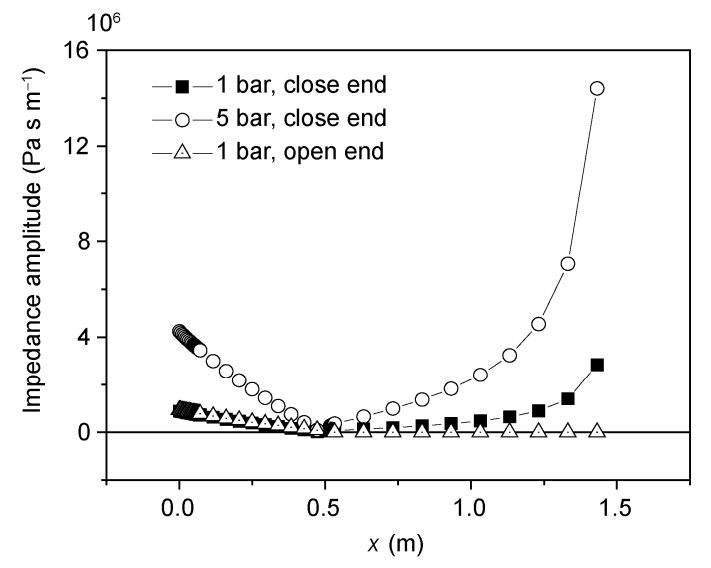

Figure 4 Impedance amplitude along the length in the closed and open acoustic configurations.

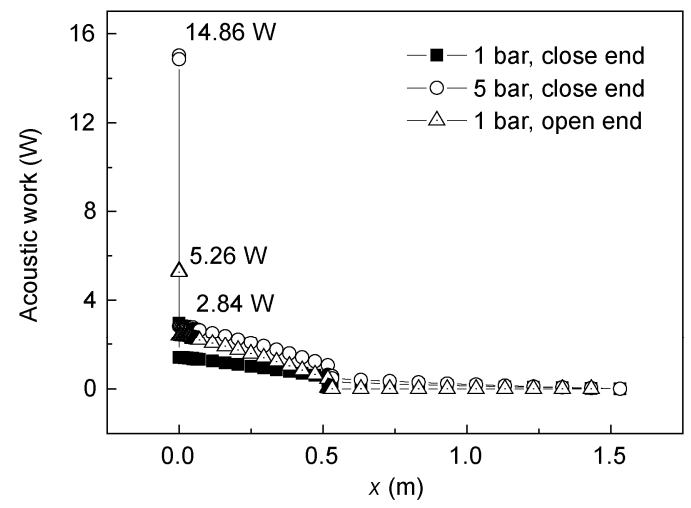

Figure 5 Acoustic work along the length in the closed and open acoustic configurations.

$\left|Z_{1}\right|$ along the interval between $0-0.53 \mathrm{~m}$ is nearly equal for both closed and open systems at a mean pressure of 1 bar air. It is helpful to fill the resonator with to a high pressure to improve $\left|Z_{1}\right|$.

The acoustic work, $\dot{E}_{2}$, done in the closed and open acoustic configurations is shown in Figure 5. It was inferred from the relationship: $\dot{E}_{2} \sim \frac{1}{2}\left|p_{1}\right|\left|U_{1}\right| \sim \frac{\left|p_{1}\right|}{p_{\mathrm{m}}} \frac{\left|u_{1}\right|}{a_{0}} \frac{p_{\mathrm{m}} a_{0} A}{2}$, and that a higher $\dot{E}_{2}$ can be acquired for higher values of $\left|p_{1}\right|$ and $\left|U_{1}\right|$. The total $\dot{E}_{2}$ produced in the open system is $5.26 \mathrm{~W}$, and $2.38 \mathrm{~W}$ is transferred into the open resonator. However, $\dot{E}_{2}$ dissipates rapidly to zero at the open end. For the closed acoustic configuration, the total $\dot{E}_{2}$ is $14.86 \mathrm{~W}$, and $2.94 \mathrm{~W}$ is transferred at 5 bar. At 1 bar, the total is $2.85 \mathrm{~W}$, and the amount transferred is $1.4 \mathrm{~W}$. In both of the closed configurations the $\dot{E}_{2}$ transported into resonator dissipates gradually to zero at the closed end.

We concluded from the above investigations that the open system has a higher $\left|p_{1}\right|,\left|U_{1}\right|$ and $\dot{E}_{2}$ relative to the closed system at the same pressure. $\left|p_{1}\right|$ and $\dot{E}_{2}$ are lower in the 1 bar open system compared with those in the 5 bar closed system. It is difficult to design an open system, because of the rapid losses in $\left|p_{1}\right|$ and $\dot{E}_{2}$ at the open end. For- tunately, acoustic radiation can be used for long-range propagation, which was not included in this model. Therefore, it is optimal for a 1/4 wavelength system to use acoustic amplified components to improve the performance of the system. Based on linear thermoacoustic equations [16] and the above analysis, an open-air traveling-wave thermoacoustic generator was designed and fabricated.

\section{Experimental setup}

The experimental setup of the open-air traveling-wave thermoacoustic generator is shown in Figure 6 and is composed of three parts: a looped tube, a resonator and a cone. The looped tube is composed of a compliance tube, inertance tube and some thermoacoustic components. These include a hot and cold heat exchangers and a regenerator. Eight piezoelectric pressure sensors for measuring the pressure amplitude are placed along the system, and are indicated as $\mathrm{P}_{1}-\mathrm{P}_{8}$ in Figure 6 . In the looped tube, the pressure drop in heat exchanger, compared with that in the regenerator, was small and negligible. Therefore the pressure, which was measured using sensor $\mathrm{P}_{1}$ as a reference, was distributed at the input of cold heat exchanger. Sensor $\mathrm{P}_{2}$ was placed at the output of the compliance tube to measure the pressure drop. Pressure sensors $\mathrm{P}_{3}-\mathrm{P}_{8}$ were used to measure pressure losses in the inertance tube, through regenerator, thermal buffer tube and resonator, respectively. In addition, measured pressures could be analyzed using the acoustic field distribution and pressure waveform. Sensor $\mathrm{P}_{9}$ was an acoustimeter with high sensitivity, which was used to acquire the acoustic pressure far away from the output of the resonator. Thermocouples $T_{1}-T_{3}$ were placed in the middle of heater and at the two ends of regenerator. They were used to simultaneously measure the temperature at the two ends of regenerator and the onset temperature of system. $\mathrm{T}_{1}$ and $\mathrm{T}_{2}$ were Ni-Cr thermocouples, and $\mathrm{T}_{3}$ was a $\mathrm{Cu}-\mathrm{Cu}$ thermocouple. The looped tube assured that the phase difference in pressure that generates the volumetric velocity near the regenerator is matched to the traveling-wave phase. The gas parcel initiates the Stirling thermal cycle within the penetration depth of the regenerator. The resonator stabilizes the resonant frequency, increases the acoustic impedance near the regenerator, and transfers the acoustic power. The cone improves the SPL at the output of system. This generator is smaller than $1 \mathrm{~m}$ in length and $0.3 \mathrm{~m}$ in height.

\section{Results and discussion}

The pressure amplitudes $\left|p_{1}\right|$, which were measured using $\mathrm{P}_{1}-\mathrm{P}_{8}$, are shown in Figure 7. During these measurements, the heating power was $210 \mathrm{~W}$. In Figure 7, it can be seen that $\left|p_{1}\right|$ was at its maximum near the cold heat exchanger. Along counterclockwise direction in the looped tube, $\left|p_{1}\right|$ steadily decreased from $\mathrm{P}_{2}$ to $\mathrm{P}_{5}$, and in the resonator from $\mathrm{P}_{6}$ to $\mathrm{P}_{8}$. The peak-to-peak pressure amplitude ( $\mathrm{P}-\mathrm{P}$ pressure 


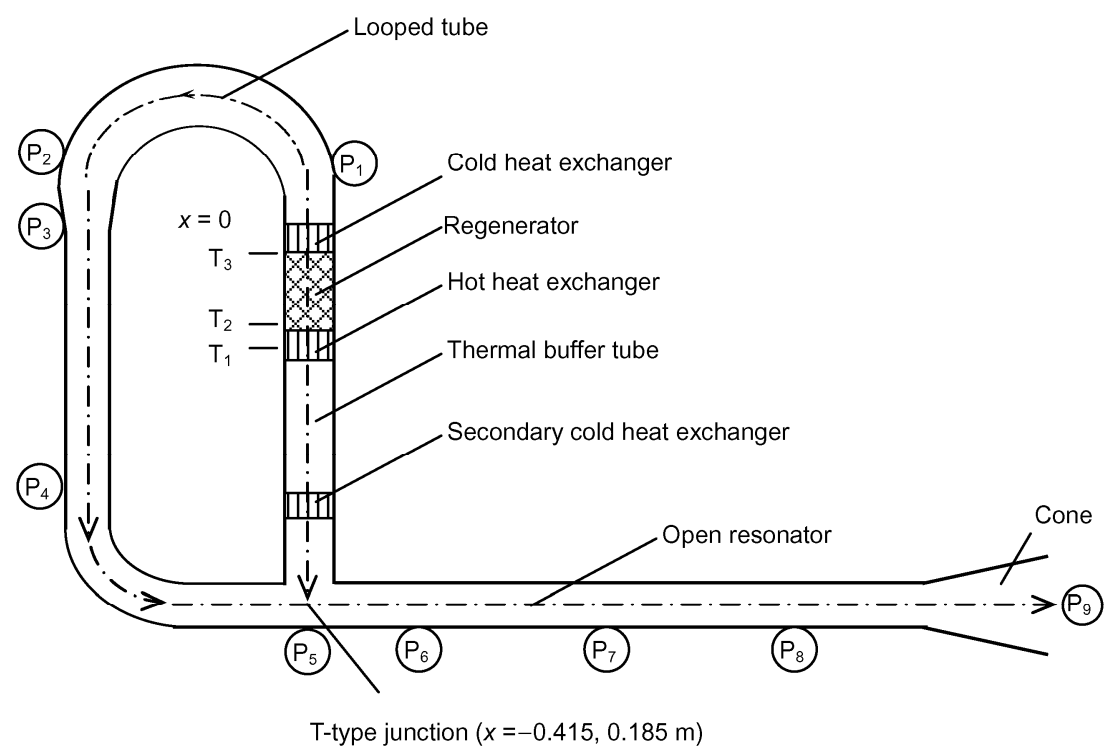

Figure 6 Experimental setup of the open-air traveling-wave thermoacoustic generator.

amplitude) at $\mathrm{P}_{1}$ was $24.06 \mathrm{kPa}$ and the $\mathrm{SPL}$ was $161.6 \mathrm{~dB}$. This was calculated by using $\mathrm{SPL}=20 \log _{10} \frac{p_{\mathrm{e}}}{p_{\text {ref }}} \mathrm{dB}$, where $p_{\mathrm{e}}$ was the measured pressure and equal to P-P pressure amplitude here, $p_{\text {ref }}$ was the referenced pressure with the value of $2 \times 10^{-5} \mathrm{~Pa}$ in the air.

The upper surface of cold heat exchanger was set at the origin of $x$-coordinate. Unfolding the looped tube in counterclockwise direction along the compliance tube, the inertance tube was at a negative position in $x$, and in the clockwise direction, the cold heat exchanger, regenerator, thermal buffer tube and resonator were at positive positions in $x$. The pressure amplitude along the length of the system was analyzed and shown in Figure 8 for various heating powers. The P-P pressure amplitude increased as the heating power increased. The resonant frequency was $74 \mathrm{~Hz}$, and the acoustic speed was $347 \mathrm{~m} / \mathrm{s}$ when the ambient air temperature was $300 \mathrm{~K}$. Therefore the wavelength of system was $\lambda=a / f$,

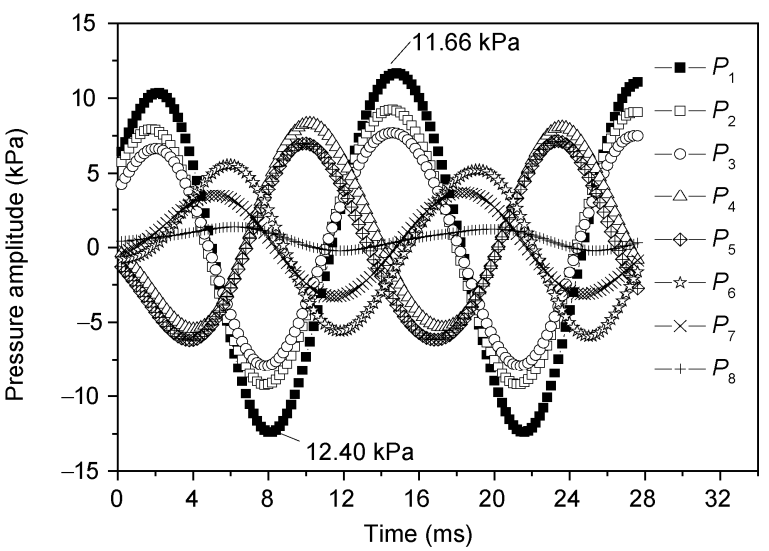

Figure 7 Pressure amplitudes versus time measured by pressure sensors $\mathrm{P}_{1}-\mathrm{P}_{8}$

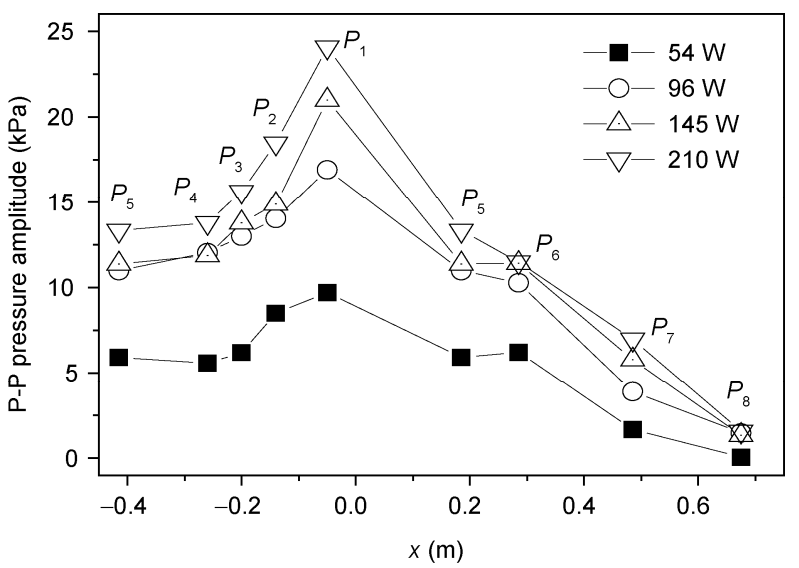

Figure 8 Peak-to-peak pressure amplitude along the length of the system measured by pressure sensors $\mathrm{P}_{1}-\mathrm{P}_{8}$.

which was $4.75 \mathrm{~m}$. The waveform of the P-P pressure amplitude was nearly $1 / 4$ wavelength.

The effect of the cone on the acoustic pressure expressed as SPL radiated from the open end of the resonator was investigated for various heating powers $Q_{\mathrm{h}}$. The results are shown in Figure 9. Without the cone, the SPL attenuated linearly with distance toward the open end of the resonator. The attenuation of the SPL could be reduced effectively using the cone for identical heating powers. In Figure 9, the results are shown with and without the cone at a heating power of $145 \mathrm{~W}$. The SPL $0.5 \mathrm{~m}$ far away from the open end of resonator was about $95 \mathrm{~dB}$, and increased nearly 15 $\mathrm{dB}$ when the cone was added. The maximum acoustic pressure at the open end and $0.5 \mathrm{~m}$ away from the open end of the resonator were $133.4 \mathrm{~dB}$ and $101 \mathrm{~dB}$ (ref $20 \mu \mathrm{Pa}$ ) for a heating power of $210 \mathrm{~W}$, respectively. The parameters, including onset temperature $T_{\text {onset, }}$ the temperature in the heater $T_{\text {heater }}$, the hot temperature $T_{\mathrm{h}}$, the cold temperature $T_{\mathrm{c}}$ and the acoustic pressure at the open end of resonator, are 
listed in Table 2 for heating powers of $145 \mathrm{~W}$ and $210 \mathrm{~W}$ with and without the cone.

Using the cone significantly decreased the onset temperature and the temperature in the heater. The absolute temperature ratio between the two ends of regenerator is defined as $\tau=T_{\mathrm{h}} / T_{\mathrm{c}}$. The acoustic power has a direct ratio with $\tau$. A lower temperature in the heater means that more heating power can be added to the system, which is important for achieving a large $\tau$. Therefore, more acoustic power and a higher SPL can be acquired with the cone. With further investigations into the configuration and the line type of the cone, the SPL at the open end of the resonator could be optimized.

\section{Conclusions}

In this paper, we designed and fabricated an open-air traveling-wave thermoacoustic generator. This design has the advantages of being small and having a simple configuration. We compared the closed and open acoustic configurations in detail. We modeled the effects on the pressure, volumetric velocity, impedance and acoustic work. We concluded that the open system has a higher $\left|p_{1}\right|,\left|U_{1}\right|$ and $\dot{E}_{2}$ relative to the closed system for the same mean pressure (1 bar air). $\left|p_{1}\right|$ and $\dot{E}_{2}$ are lower in the 1 bar open system than in the 5 bar closed system. In the open system the modeled waveform had a $\left|p_{1}\right|$ of a $1 / 4$ wavelength system in which $\left|p_{1}\right|$ and $\dot{E}_{2}$ dissipated rapidly at the open end. Based on the above analysis, we fabricated a 1 bar open-air thermoacoustic

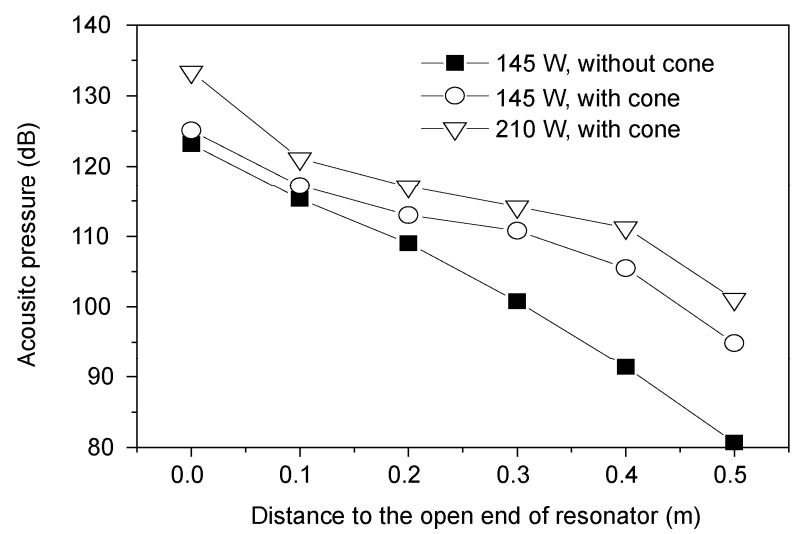

Figure 9 Acoustic pressure versus the distance to the open end of the resonator.

Table 2 Operating parameters, temperature and acoustic pressure

\begin{tabular}{ccccccc}
\hline \multirow{2}{*}{ Cone } & $\begin{array}{c}\text { Heating power } \\
\text { (W) }\end{array}$ & \multicolumn{4}{c}{ Temperature $\left({ }^{\circ} \mathrm{C}\right)$} & Acoustic pressure \\
\cline { 3 - 6 } & & Onset & $T_{1}$ & $T_{2}$ & $T_{3}$ & $(\mathrm{~dB})$ \\
\hline \multirow{2}{*}{ Without } & 145 & 429.5 & 658.8 & 371.3 & 34.8 & 123.2 \\
\multirow{3}{*}{ With } & 145 & 425 & 547.3 & 303.8 & 26.9 & 125.1 \\
& 210 & 425 & 647.8 & 348.1 & 28.2 & 133.4 \\
\hline
\end{tabular}

generator. It had a maximum acoustic pressure of $161.6 \mathrm{~dB}$ near sensor $\mathrm{P}_{1}$ at the cold heat exchanger. The cone at the open end of the resonator reduces the onset temperature and the temperature in the heater. This increased the absolute temperature ratio and acoustic pressure. The maximum acoustic pressures at the open end and $0.5 \mathrm{~m}$ from the open end of the resonator were $133.4 \mathrm{~dB}$ and $101 \mathrm{~dB}($ ref $20 \mu \mathrm{Pa})$ for a heating power of $210 \mathrm{~W}$, respectively. This is suitable for practical use as a low-frequency acoustic source. In future work, we hope to achieve an SPL of $150 \mathrm{~dB}$. At this level, the device could be a possible solution for some of the problems of existing acoustic generators, which include low acoustic pressures and system complexity. We believe this system can be used as a basic acoustic source for low frequency and long-range noise experiments, and as a supply for high acoustic pressures for studying industrial sources and vibrations.

This work was supported by the National Natural Science Foundation of China (50806081).

1 Swift G W. Thermoacoustic engines. J Acoust Soc Am, 1988, 84: 1145-1180

2 Feldman K T. Review of the literature on Sondhauss thermoacoustic phenomena. J Sound Vib, 1968, 7: 71-82

3 Wheatley J C, Hofler T, Swift G W, et al. An intrinsically irreversible thermoacoustic heat engine. J Acoust Soc Am, 1983, 74: 153-170

4 Ceperley P H. A pistonless Stirling engine-the traveling wave heat engine. J Acoust Soc Am, 1979, 66: 1508-1513

5 Yazaki T, Maekawa T, Tominaga A, et al. Traveling wave thermoacoustic engine in a looped tube. Phys Rev Lett, 1998, 81: 3128-3131

6 Backhaus S, Swift G W. A thermoacoustic Stirling heat engine. Nature, 1999, 399: 335-338

7 Gardner D L, Swift G W. A cascade thermoacoustic engine. J Acoust Soc Am, 2003, 114: 1905-1919

$8 \mathrm{Hu} \mathrm{Z} \mathrm{J,} \mathrm{Li} \mathrm{Q,} \mathrm{Li} \mathrm{Q,} \mathrm{et} \mathrm{a1.} \mathrm{A} \mathrm{high} \mathrm{frequency} \mathrm{cascade} \mathrm{thermoacoustic}$ engine. Cryogenics, 2006, 46: 771-777

9 Radebaugh R. A review of pulse tube refrigeration. Adv Cryo Eng B, 1990, 35: 1025-1191

10 Jin T, Chen G B, Shen Y. A thermoacoustically driven pulse tube refrigerator capable of working below 120K. Cryogenics, 2001, 41: 595-560

11 Sun D M, Marc D, Guenter T, et al. Investigation on regenerator temperature inhomogeneity in Stirling-type pulse tube cooler. Chinese Sci Bull, 2009, 54: 986-991

12 Zhou G, Li Q, Li Z Y, et al. Influence of resonator diameter on a miniature thermoacoustic Stirling heat engine. Chinese Sci Bull, 2008, 53: 145-154

13 Hu J Y, Luo E C, Li S F, et al. Heat-driven thermoacoustic cryocooler operating at liquid hydrogen temperature with a unique coupler. J Appl Phys, 2008, 103: 104906

14 Backhaus S, Tward E, Petach M. Traveling-wave thermoacoustic electric generator. Appl Phys Lett, 2004, 85: 105-107

15 Luo E C, Wu Z H, Dai W, et al. A 100W-class traveling-wave thermoacoustic electricity generator. Chinese Sci Bull, 2008, 53: 14531456

16 Swift G W. Thermoacoustics: A Unifying Perspective for Some Engines and Refrigerators. Sewickley, PA: Acoustical Society of America Publishers, 2002

17 Slaton W V. An open-air infrasonic thermoacoustic engine. Appl Acoust, 2010, 71: 236-240

Open Access This article is distributed under the terms of the Creative Commons Attribution License which permits any use, distribution, and reproduction in any medium, provided the original author(s) and source are credited. 